\title{
Experimental study on flame propagation characteristics of Hydrogen premixed gas in gas pipeline
}

\author{
Danzhu Ma ${ }^{1 *}$, Zhuang Li ${ }^{l}$, Fengrui Jia ${ }^{1}$, Zhou $\mathrm{Li}^{1}$ \\ ${ }^{1}$ Liaoning Shihua University, Fushun, Liaoning 113001, China
}

\begin{abstract}
Hydrogen is the cleanest high-energy gas fuel, and also is the main industrial material. However, hydrogen is more explosive and more powerful than conventional gas fuels, which restricts its application. In particular, the expansion of premixed combustion under a strong constraint is more complicated, the reaction spreads faster. The flame propagation characteristics of premixed hydrogen/air were investigated by experiment. The mechanism of reaction acceleration is discussed, and then the speed of the flame propagation and the reaction pressure were tested and analysed.
\end{abstract}

\section{Introduction}

Flammable gas refers to the gas that can cause combustion or explosion and release a large amount of energy under the ignition energy excitation within the environment full of combustion improver. The flammable gases play a very important role in the fields of petrochemical industry, metallurgical industry and gas production and supply industry, such as methane and hydrogen. By far, hydrogen is the cleanest high-energy gas fuel, and also is the main industrial material. However, hydrogen is more explosive and more powerful than conventional gas fuels due to the lower ignition energy, the wider explosion limitation and the larger laminar burning and diffusion velocity. The hydrogen explosion accidents occur frequently. The research on the combustion and flame propagation characteristics of hydrogen premixed system has a great practical significance. The propagation of gas explosion wave, the kinetic theory of laminar flame and the constant flame theory were discussed in the early 1950s [1-2]. The propagations of gas explosion wave in the closed system were also focused as follow [3-6]. But the investigations about the hydrogen premixed system are not enough. It has very important practical significance and is the key of whether hydrogen can be used as a conventional energy.

In this paper, the deflagrating characteristic of premixed hydrogen was concerned, based on the theory of flame dynamics and pressure change. The speed of flame propagation and the pressure fluctuation were studied and analysed.

\section{Hydrogen premixed combustion and its mechanism in the constrained space}

The combustion and explosion of premixed gas needs the conditions, such as the suitable flammable gas concentration, enough oxygen and ignition source. It is a process of chemical reaction flow. The typical process of premixed flame propagation in the constrained space includes the ignition, the laminar combustion, the fire perturbation, the transformation from laminar to turbulent combustion and the final detonation. At the beginning of the reaction, the laminar flame is unstable. The fold appears caused by the instability of the thermal diffusion perturbation. The vortex generated to increase the disturbance of the flame front and to prompt the turbulent combustion, when the explosion space is constrained or there are obstacles. Then, if the flame continues to accelerate, the explosion occurs and even transforms to detonation. Figure 1 gives a sketch of the propagation process of the reaction.

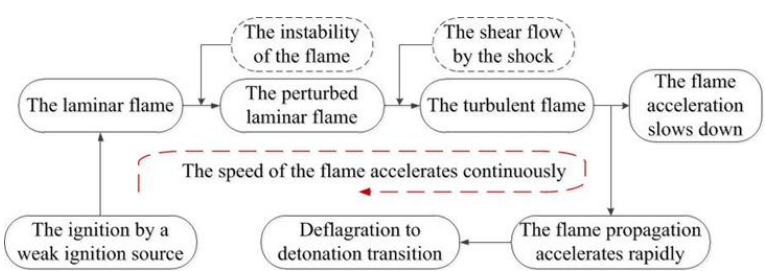

Fig. 1. A sketch of the propagation process of the reaction.

The following combustion chemical reaction occurs, when premixed hydrogen is ignited.

$$
\mathrm{H}_{2}+0.5 \mathrm{O}_{2}+1.88 \mathrm{~N}_{2} \rightarrow \mathrm{H}_{2} \mathrm{O}+1.88 \mathrm{~N}_{2}
$$

In this paper, four different hydrogen concentrations were considered for test, they are $13.5 \%, 18 \%, 24 \%$, $28.5 \%$ and $38.5 \%$.

\section{Experimental system}

The experimental setup is shown as in figure 2, which consists of an automatically gas supply system, a high explosive pressure test system, a high-speed photography system, a high-voltage ignition system and a pressure relief channel. The simulated pipe used in the

*Corresponding author: danzhuma@163.com 
experiment was made by polymathic methacrylate tube. The inner diameter of the tube was $108 \mathrm{~mm}$, with a uniform length of $2 \mathrm{~m}$. Two relief channels are set on the side of the pipeline to ensure the safety. The ignitions were charged by high voltage spark discharge. The reaction pressures and the flame propagation were recorded by the pressure sensors and high-speed camera.

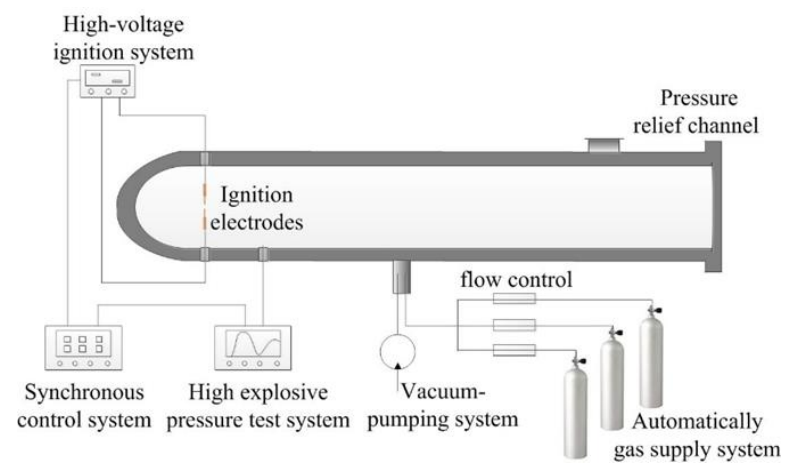

Fig. 2. Schematic of the experimental setup.

\section{Results and discussion}

\subsection{The speed of flame propagation of premixed hydrogen in the pipeline}

The reaction wave fronts were recorded by the highspeed camera, and then the speed of the flame propagation could be calculated by the image processing method. The position of flame front is calculated and the speed of propagation is the ratio between the distance and the time difference between two positions, as shown in figure 3. In this paper, the speed of flame propagation under different hydrogen volume fraction is calculated by using the above method.

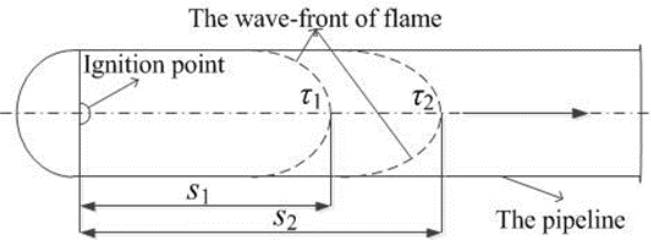

Fig. 3. The sketch of the flame propagation in the pipeline.

The effect of different hydrogen volume fractions on the speed of flame propagation was discussed. The results are shown in figure. 4 . The propagation speed of the flame in the pipe increased with the flame front propelling, and then decreased after the peak for $X_{\mathrm{H}_{2}}=13.5 \%$ and $X_{\mathrm{H}_{2}}=18 \%$. The speed of flame propagation decreased with the irregular flame front. But the decrease of the speed was not tested in the higher hydrogen content.

The peak speed of flame propagation and the location of the peak are all related to the hydrogen concentration. The higher the concentration of hydrogen, the more violent the reaction is, the faster the flame spread. The propagation speed of the premixed gas in the restricted pipeline is closely related to the shape of the flame front. In the early stage, the flame is not restrained by the wall, it is semi-spherical and rapidly evolves into a fingertip flame, the surface area of the flame is accelerated by exponential growth. When the flame comes into contact with the pipe wall, the acceleration is terminated into the plane flame phase, the propagation speed of the flame front is beginning to decline. Then the flame forward reverses, gradually forming the tulip flame, which increases the surface area of the flame, the flame forward speed increases again. It can be seen from figure 4 that the tulip flame appears at $X_{\mathrm{H}_{2}}=18 \%$. But, at the higher hydrogen concentration, the premixed flame is in the accelerated propagation period, there is no feature of tulip flame in the length of the experimental pipeline.
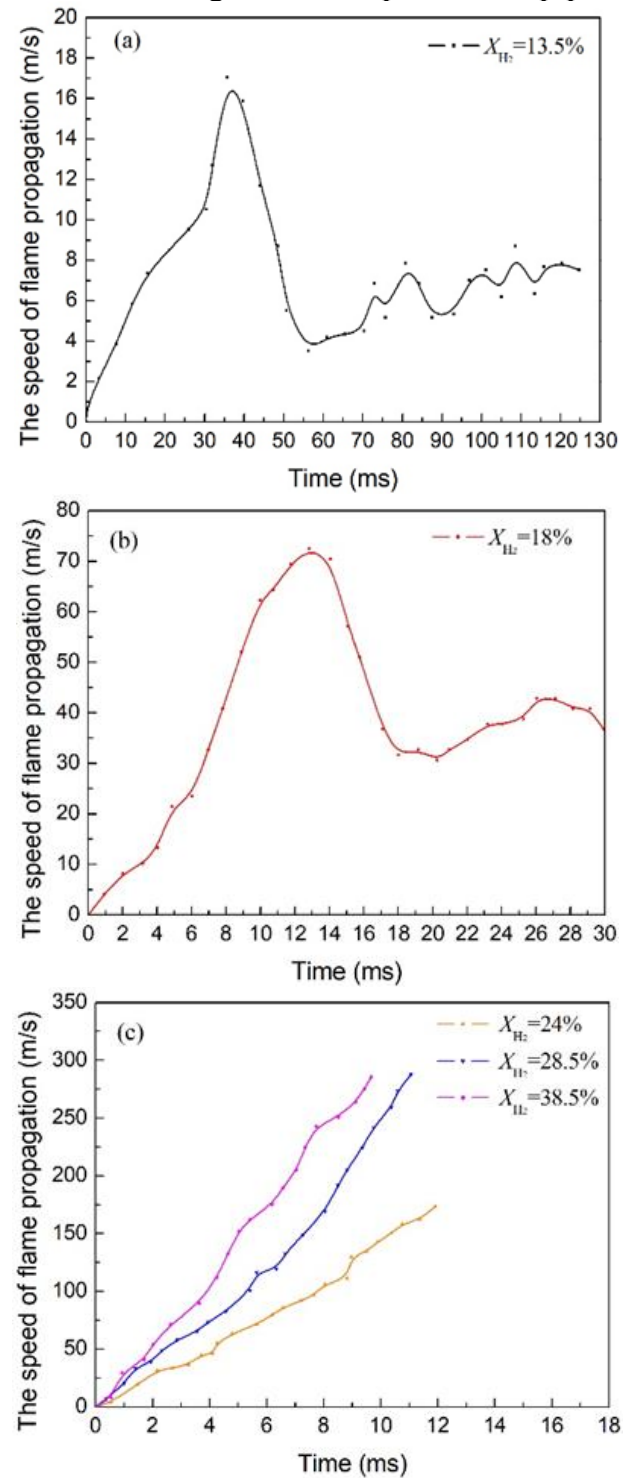

Fig. 4. The flame propagation speed curves of pipelines with different hydrogen concentration.

\subsection{The reaction pressure}

The reaction pressure was recorded by the pressure sensor, which was lie in the front of the pipe. The typical curves were shown in figure 5. As shown in the figure, the reaction pressure of premixed hydrogen-air has obvious pressure oscillation and appears negative pressure. At the beginning of the reaction, the pressure increases rapidly with the reaction progresses. Then the film on the other side of the pipeline breaks, the pipe becomes to a semi-enclosed space, part of the premixed 
gas washes out of the pipe. At the same time, the gas in the pipeline continues to participate in the reaction and consumed, the pressure drops in the pipe, resulting the negative pressure. The negative pressure in the tube causes the ejected gas to be sucked back into the reaction, causing the pressure rise again, thus causing the pressure oscillation. With the constant consumption of unburned gas in the tube, the absolute value of the pressure oscillation decreases and finally returns to the initial value.

The results of the pressure test show that the pressure propagation of hydrogen-air in the pipeline is divided into three stages. (1) After the input of the ignition energy, the premixed gas is ignited, the hydrogen-air continue to react, the pressure in the pipe continuously accumulates to reach the first crest value. (2) Due to the high temperature of the premixed combustion, when the water vapor which generated by the reaction contact the lower temperature environment to condense into droplets. This process causes the negative pressure in the pipe. And then, the unreacted hydrogen-air are sucked back to the pipe to join in the reaction, the pressure goes up again. This causes the pressure shock in the pipeline. (3) The pressure amplitude reduces to the initial value with the complete reaction of the premixed gas. The peak pressure and the intensity of the pressure oscillation increase with the increasing of hydrogen content, while the time of peak pressure decrease.

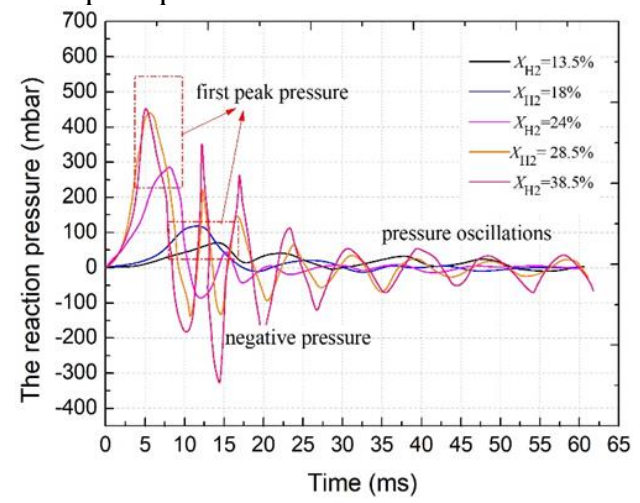

Fig. 5. Typical reaction pressure curve.

\section{Summary}

The combustion characteristics of premixed hydrogen is the foundation of its safe application as a high-energy fuel. The experiment results show that the peak speed of flame propagation and the location of the peak depend on the concentration of hydrogen. The higher hydrogen concentration, the more violent the reaction is, and the faster the flame spread. The reaction pressure develops through three stages: the pressure rises to the first peak; the pressure continues to oscillate and the pressure reduces to the initial value.

\section{Acknowledgements}

This work was financially supported by the Scientific Research Project of the Department of Education of Liaoning Province (L2015303) and the Breeding program of Liaoning Shihua University (2016[177]-19).

\section{References}

1. W. Bartknecht, Explosions: Course, Prevention, Protection (Berlin, Springer, 1981)

2. D. Bjerketvedt, J. R. Bakke, K. Wingerden, Gas Explosion Handbook (Journal of Hazardous Materials, 1997)

3. K. K. Kuo, Principles of Combustion (WileyInterscience, Switzerland, 2005)

4. Mustafa Ilbas, Serhat Karyeyen, Ismail Ozdemir, Int. J Hydrogen energy, E 26, 1 (2015)

5. Xianzhong $\mathrm{Hu}$, Qingbo $\mathrm{Yu}, \mathrm{Nan}$ Sun, Qin Qin, Int. J Hydrogen energy, E 39, 9527 (2014)

6. Hongfa Huo, Xingjian Wang, Vigor Yang, Combustion and Flame, E 161, 3040 (2014) 